\title{
Deep neural network enabled fault detection in LVDC microgrid using empirical mode decomposition
}

\author{
Dipti Patil $^{1 *}$, Bindu $S^{2}$ and Sushil Thale ${ }^{3}$ \\ Research Scholar, Department of Electrical Engineering, Fr. C. Rodrigues Institute of Technology, Maharashtra, \\ India $^{1}$ \\ Professor and HOD, Department of Electrical Engineering, Fr. C. Rodrigues Institute of Technology, Maharashtra, \\ India $^{2}$ \\ Professor and Dean, Department of Electrical Engineering, Fr. C. Rodrigues Institute of Technology, Maharashtra, \\ India $^{3}$
}

Received: 05-October-2021; Revised: 11-February-2022; Accepted: 14-February-2022

(C2022 Dipti Patil et al. This is an open access article distributed under the Creative Commons Attribution (CC BY) License, which permits unrestricted use, distribution, and reproduction in any medium, provided the original work is properly cited.

\begin{abstract}
An advanced method of fault recognition and location identification for a low voltage direct current (LVDC) microgrid is introduced in this paper. Sources are tied-up with power electronics converters to the microgrid. Components of converters are very much at risk of damage during a fault. It became necessary to isolate the healthy part fast. Again, magnitude of fault current increases at a very high rate during the fault, the entire system might get de-energized and making it difficult to identify the fault location. With a view to this, an empirical mode decomposition (EMD) based deep learning classifier is proposed to recognize and locate the fault in the LVDC microgrid. EMD based methods are suitable for a system where transient values of the fault current are an important feature to detect a fault. EMD decomposes the fault signal into different components to detect the temporal variation (transient caused in segment current because of the fault). Convolutional neural network (CNN) the architecture of deep neural network $(D N N)$ is used to classify the signal into normal and faulty, as well as to recognize the fault location in the system. The suggested technique is evaluated through MATLAB/Simulink simulations. The data set needed for the classification of normal, fault, and abnormal condition signals are collected under different fault resistances, fault locations, and various system conditions. The outcome shows that the classifier has a high accuracy of $94.97 \%$ and a low error rate compared to other classification models such as Gaussian mixture model (GMM) and support vector machine (SVM). The method is also validated on designed and developed laboratory ring-type LVDC microgrid hardware. Texas Instruments' TMS320F28069 digital signal processors (DSP) are employed for the execution of the proposed protection scheme. The hardware result also demonstrates the proposed methodology's superior performances and gives an accuracy of $90.60 \%$.
\end{abstract}

\section{Keywords}

Fault detection and protection, DC microgrid, Empirical mode decomposition, Convolutional neural network, Deep neural networks.

\section{Introduction}

Direct current (DC) microgrids are small-scale distribution systems comprising distributed generators, storage instruments, and controllable loads operating in a coordinated way [1]. Power electronic interface utilized to attach sources and a load to the DC microgrid, which provides flexible control and improved power quality. It makes the microgrid single controlled units that meet the local energy needs and can also operate in coordination with the main grid or utility power system.

*Author for correspondence
DC microgrids require fewer power electronics converters compared to alternating current (AC) systems.

The reactive power loss and harmonics are absent in the case of the DC microgrid and it does not require synchronization [2]. Even though the dominance of DC microgrids is significant, the protection of DC microgrids has achieved numerous obstacles [3]. Because of the presence of numerous sources and due to the introduced energy storage system (ESS) permits power stream both ways in DC microgrid. Also, topological changes in the network due to connection or disconnection of generators, storage 
systems, and loads are responsible for a distinct fault current magnitude [4]. So, the regular over-current and nondirectional relay for a specific fault condition make an operational error in other fault types. Generally, In a DC microgrid, the load and converters are associated at the load side and work in closed-loop control, acting as a constant power load. During the fault condition voltage begin to diminish quickly and to keep up with steady power, this constant power load draws enormous current which essentially sets up incremental negative resistance [5]. When DC microgrid operates in standalone mode the constant power load promotes voltage and current oscillations, which additionally create an enigma for protection relays to distinguish between fault condition and system oscillations [6]. Along with this, one of the significant difficulties is the rapid rise of fault current in a short duration because of the quick release of DC-link capacitors. The low impedance of the line further expands this peculiarity. For that reason, the fault must be correctly detected and isolated as quickly as possible for costly loads and power converter's security [7].

In this way, while DC microgrid gives critical benefits as far as adaptability and durability, the execution of DC microgrid is subverted because of the inescapable difficulties that emerge because of protection. Therefore, this paper is dedicated to the development of advanced and fast protection schemes that will be suitable for the reliable and fast protection of DC microgrids. The fault detection is based on fault current characteristics appearing at the time of the fault. Each type of fault will have distinct characteristics depending on fault location, fault resistance, and system condition. The proposed methods learn these characteristics during training and compare them with unknown fault signals during testing. Hence method assures the fast and accurate fault detection and location identification.

The paper is arranged as follows; the literature review and research gaps in it are discussed in section 2 . The proposed protection methodology is explained in section 3. The developed MATLAB simulation model and DC fault current analysis are also discussed in this section. Section 4 covers the simulation results of the proposed technique and comparison with existing classifier methods. The development of a laboratory prototype of a low voltage direct current (LVDC) microgrid and experimental results to authenticate the suggested protection method are also discussed. Discussion and limitations as well as the complete conclusion from the paper have been conveyed in sections 5 and 6 .

\section{Literature review}

Fault detection and localization in DC microgrid can normally be realized by traveling-wave or injectionbased algorithms. However, the small length cables of the DC microgrids make it hard to compute the time difference using traveling waves with sufficient precision. Also, the non-appearance of frequency and phasor information in DC systems place restrictions on the utilization of this method [8]. Non-unit protection of the fault has been identified by investigating the current, voltage, $d i / d t, d v / d t$, and impedance variation during the fault. However, the acuteness of the non-unit protection technique is based on the righteousness of values and appropriate time interval [9]. Unit type protection is active in a zone and it does not acknowledge an external circuit fault, also these methods require synchronized data over communication links [10]. Multiple algorithm techniques which have been specified in literature to identify the fault type and location are very complex. The proposed method relies on the magnitude of fault current and voltage sample and it does not consider the dynamics of the system [11]. In recent years data-driven and digital signal processing approach to detect the fault in DC microgrids becoming more popular. These techniques have high computation speed and satisfactory fault classification potential in real-time. Additionally, discrete Fourier transform and discrete wavelet transform (DWT) like digital signal processing techniques normally use initialized input signals to bring out the time-frequency features for the investigation. Nevertheless, these methods cannot provide fault-type information [12]. The artificial neural network technique is proposed by Leh et al. [13] for power transmission line, but the analysis shows low noise handling capability. Chaotic neural network and wavelet transform based fault detection method is presented by Wang and $\mathrm{Xu}$ [14] but this method increases the computation burden as well as complexity in fault detection. Ashok and Yadav [15] proposed the maximal overlap discrete wavelet transform (MODWT) based algorithm for fault classification which is very computational intensive. Fault detection and classification scheme proposed by Paul and Debnath [16] uses wavelet features and fuzzy based classifier, here results show that fuzzy does not give high classification accuracy.

Lack of awareness and technical guidance, the low inertia of the system, bidirectional power flow, 
topological changes, and the constant power load reduces the efficacy of traditional conventional overcurrent and under-voltage protection techniques. To cope with that many protection techniques is available in the literature but have some shortcomings as discussed above. This paper proposed empirical mode decomposition (EMD) based deep neural network (DNN) classifier for fault recognition and location identification in an LVDC microgrid. The EMD extracts the characteristics of the fault signal and these extracted features are utilized at the time of training of the neural network. Higher accuracy is the advantage of feature extraction-based fault detection. This method classifies the fault based on features collected from electrical measurement in various fault scenarios. Hence, this technique is autonomous of system factors like topology and line parameters, it will have an inherent implementation possibility when utilized in fault detection and identifying the location within the scheduled time frame.

A small-scale ring-type LVDC microgrid simulation and hardware implementation are planned and evolved to conduct the recommended study. DC system current and the voltage signal are measured under usual and fault conditions to examine the fault characteristics. The convolutional neural network (CNN) training set is built by creating a fault in the DC microgrid at different locations with nonidentical fault resistance. Following the fault detection, the faulty segment will be cut off from the healthy system and normal operation is restored.

\section{Methods}

\subsection{Proposed protection methodology}

In the DC microgrid system, generally the smaller number of measuring units are provided and a low sampling rate is embraced, it provides finite data for fault detection. The proposed methodology uses large data collected from the DC microgrid for various fault scenarios and abnormal conditions. DNN learns the features automatically and identifies the primary data. It shows promising capability in discriminating the fault or abnormal conditions in the DC microgrid. Additionally, the introduction of EMD for fault feature extraction before feeding fault signal to CNN improves the accuracy of fault detection.

The proposed protection method is shown in Figure 1. The segment currents and bus voltage are measured by placing relays at both ends of all segments of the DC microgrid. These segment currents and bus voltage are acting as the data set which contains all information about the operating condition of the system. The collected data set is preprocessed first and then given to EMD to extract the fault features. These extracted fault features are used to train and test the $\mathrm{CNN}$ classifier. $\mathrm{CNN}$ classifier will identify the fault location. The DNNs are constructed with TensorFlow.

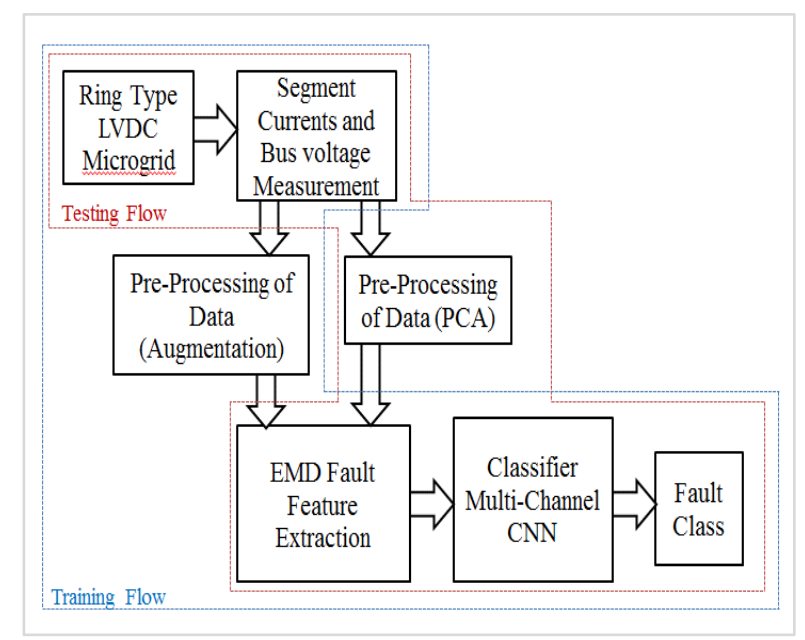

Figure 1 Block diagram of proposed methodology

\subsection{Type of data set}

The data set required to feed to the algorithm is collected for different system conditions like normal, overload, over-voltage, light load, and fault type 1 to $\mathrm{N}$ and labeled accordingly. Fault type 1 to $\mathrm{N}$ is the bus to bus fault is created at dissimilar locations with non-identical fault resistance in each segment. The relays placed at both ends of each segment will provide information on segment current and bus voltage. The normal, fault type 1 to $\mathrm{N}$, overload, over-voltage, and light load signal parameters constitute the column of stored data. The rows are samples of signals depending on simulation time and the sampling frequency.

\subsection{Augmentation and pre-processing of Data 3.3.1Data augmentation} The augmentation techniques help to create synthetic data by transforming existing labeled data to help the model span the intra-class variances which data can have [17]. Data augmentation is done by adding noise into the actual signal. In practical cases, the fault signals do get affected by measurement, system, and electromagnetic interference noise. Ten different fault signals are generated from the single fault measurement by adding random noise of zero-mean Gaussian distribution. This expands the dataset by ten times. Along with that different amount of smoothing 
and sharpening is applied to the fault signal. For experimentation 2nd and 3rd order smoothing and sharpening are used to increase the data size by 4 times. Effectively for training $40(10 \times 2 \times 2$ : random $\mathrm{x}$ smoothing and sharpening) times, the measured data is generated.

\subsubsection{Pre-processing}

The pre-processing of the dataset is done by principal component analysis (PCA). PCA is a self-governed investigating algorithm that helps in dimensionality reduction of the original data in machine learning. This investigation is established on a linear transformation that produces new uncorrelated variables (components) from the primary correlated measured variables. It is a technique to draw a few of these components that are sufficient to adequately represent the hidden sources of variability in the process. PCA works by examining the discrepancy of each feature because the high feature shows a good split between the classes, and in consequence, it decreases the dimensionality [18]. EMD is then applied to pre-processed data to extract the important fault features before using the same data to train the CNN.

\subsection{Empirical mode decomposition (EMD)}

The EMD method extracts low-frequency structures and represents a signal like the multi-resolution analysis. EMD allows the data to be examined in an adaptive time-frequency-amplitude space for nonstationary signals [19]. It decomposes the input signal into a user-defined number of intrinsic mode functions (IMF). The successive extremes of the input signal are recognized first, then every local maxima is linked by some interpolation technique to get an upper envelope, and similarly, the procedure applies to get the lower envelope. The mean trend is calculated from these two envelopes. The variance between the mean trend and the input signal is the first IMF component. Then in the next iteration the first IMF is treated as an input signal and the same steps are followed to get the next IMF component. EMD decomposition breaks the signal in a multistage structure and each component contains a different band of frequency. The EMD of segment currents and the bus voltage data set are taken separately for the analysis [20].

\subsection{Convolutional neural networks (CNNs)}

CNNs are built up of neurons that have learnable weights and biases. As shown in Figure 2, fault signals fed to CNN go through multiple convolutional and pooling layers. In case of signal classification, the last layer of $\mathrm{CNN}$ is a dense layer.
This layer receives input vector which is then mapped to a new output vector having a dimension equal to types of faults [21]. It is essential to examine fault signal information estimated at different areas in the DC microgrid and required to select the correct pre-handling and artificial intelligence

(AI) computation [22].

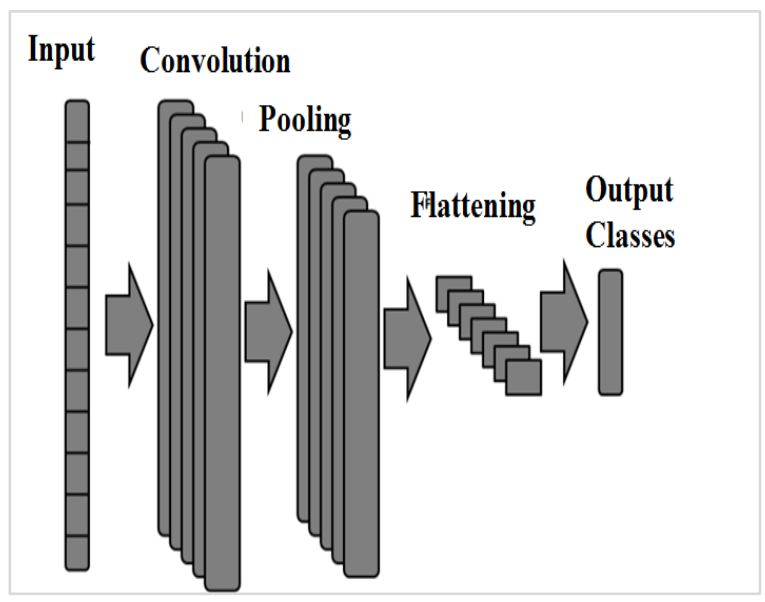

Figure 2 Architecture of 1D CNNs

3.6DC microgrid simulation model

A single line ring-type four-segment 48 volt DC low voltage microgrid shown in Figure 3 is modeled in Matlab (Figure 4) [23]. The $1 \mathrm{~km}$, bus segment consists of a $10 \mathrm{~m} \Omega$ resistance also, $100 \mu \mathrm{H}$ inductor and the line stray capacitances were disregarded. The system is built with two photovoltaic (PV) sources, one energy storage system, and the load. The PVs are linked to the DC bus through DC-DC converters, operated under maximum power point tracking (MPPT) mode shown in Figure 5. The DC battery with the bidirectional DC-DC converter worked as a voltage source converter (VSC) and operated with state of charge (SOC) controller as shown in Figure 6 [24].

During normal operation, once the battery is fully charged, then it works as a backup power supply. However, in the case of solar energy unavailability, battery energy acts as the stiff voltage source to maintain the system under steady-state conditions. DC loads are straightforwardly associated with DC microgrids. Throughout the DC line fault, the circuit breakers connected at one and another end of the segments operate and disconnect the faulty segment, and keep up with stable activity with no power outage. After the disconnection of the faulty segment, the load is met by the remaining part of the network by increasing its transmission capacity [25]. The 
practicable faults that exist in the DC microgrid system are DC bus-to-bus and bus-to-ground faults. The bus-to-bus fault is observed as the permanent fault and is considered in this paper. During faults, an absolute route will appear through the antiparallel diode in the VSC, and converter stations will deliver active power to the occurred fault [26]. This may result in the high increase of current in the DC segment and the change of current direction. The bus to bus fault is created in segment 1 at $10 \%$ and $90 \%$ distance of the 1-km bus segment. Figure 7 shows the segment fault current estimated from the two closures of the segment. The fault current is having a fast-rising transient with a large peak followed by oscillation. The segment fault currents measured at both ends of the segment by embedding diverse fault resistance $(0.1 \Omega$ and $0.9 \Omega)$ at a half distance of a 1 $\mathrm{km}$ bus segment are shown in Figure 8 . The rate of rise of the fault current and magnitude changes with respective fault location and fault resistance. These fault current features are used in the suggested technique to identify the location of the fault in the segment and feed as an input to the designed DNN to classify.

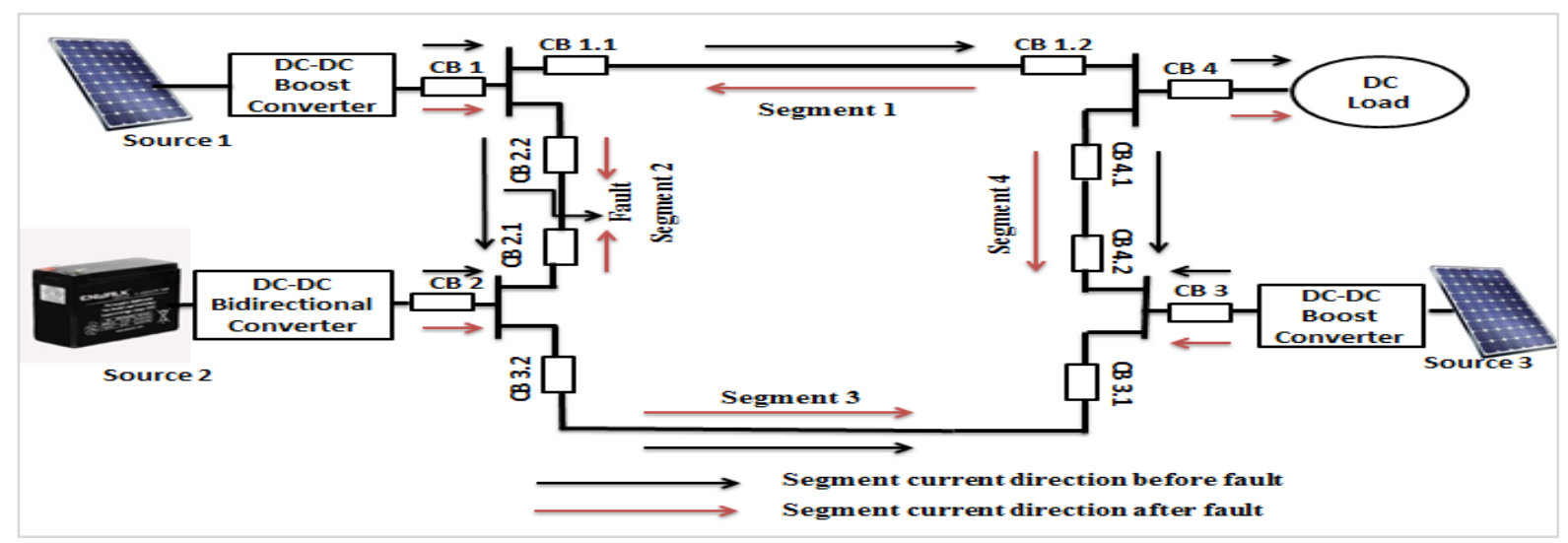

Figure 3 Single line diagram of 48 V LVDC Microgrid

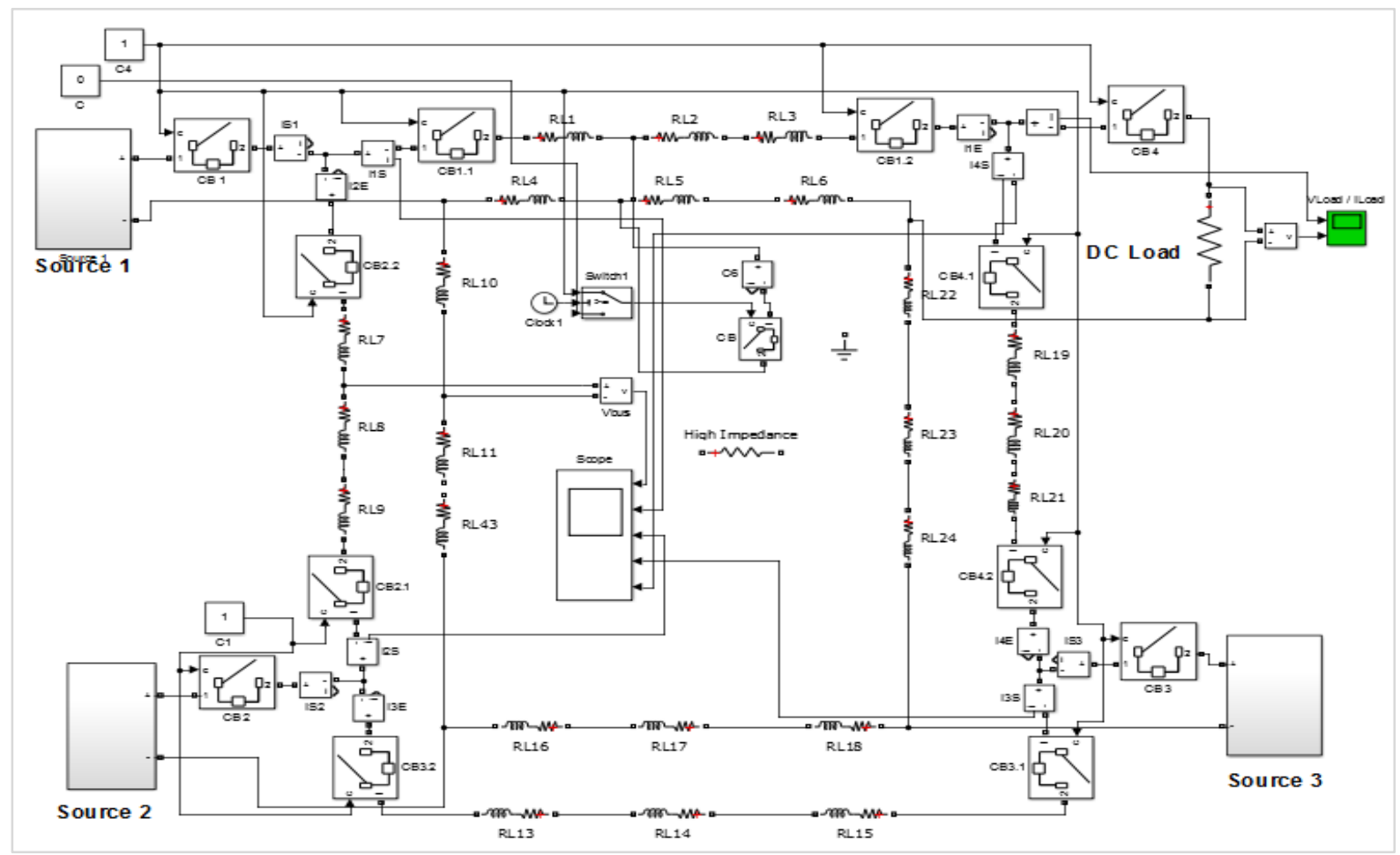

Figure 4 Simulation model for 48 V DC Microgrid 


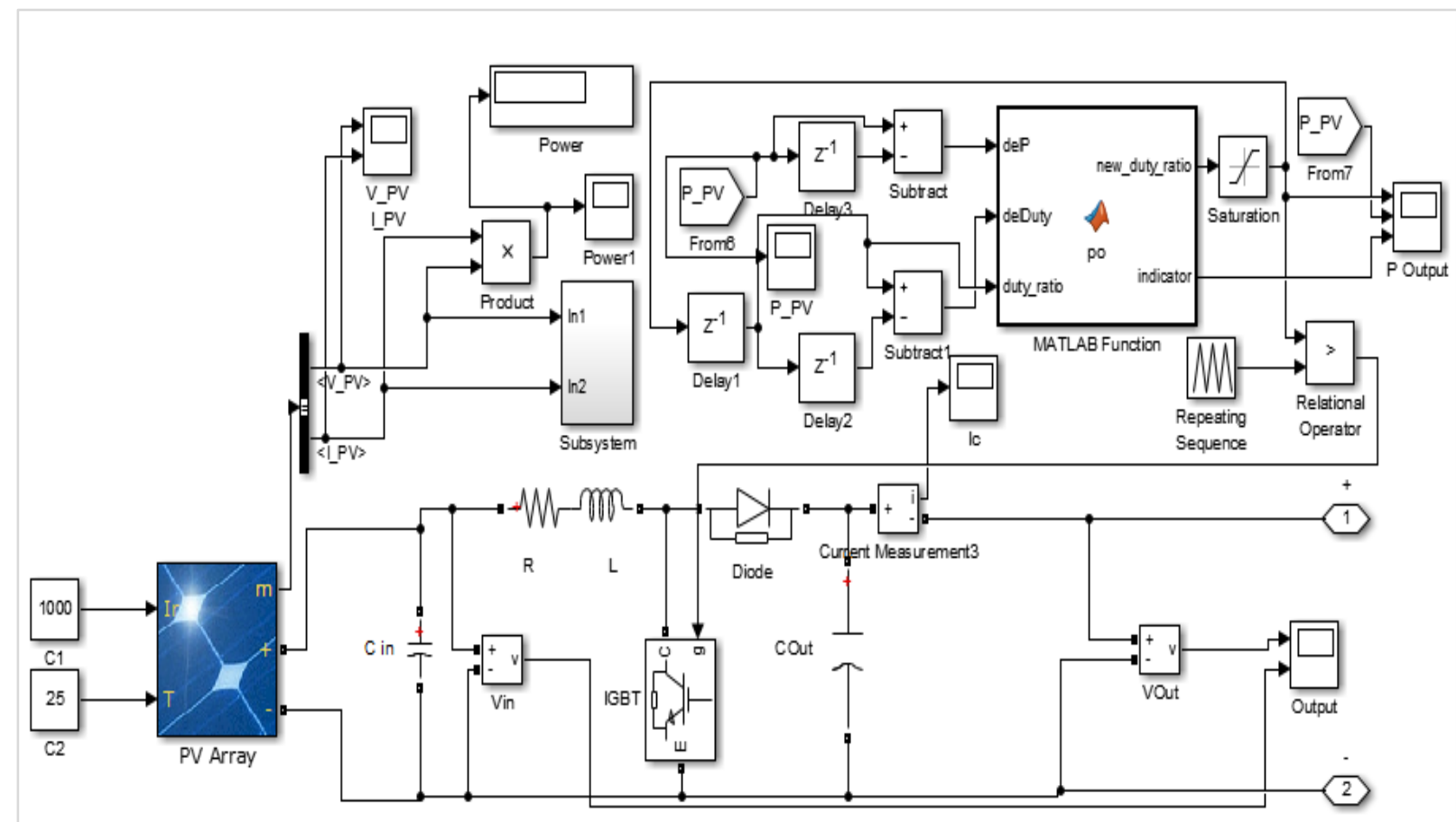

Figure 5 Simulation model for source 1 and source 3 -PV source associated with DC microgrid across DC-DC boost converter with MPPT controller

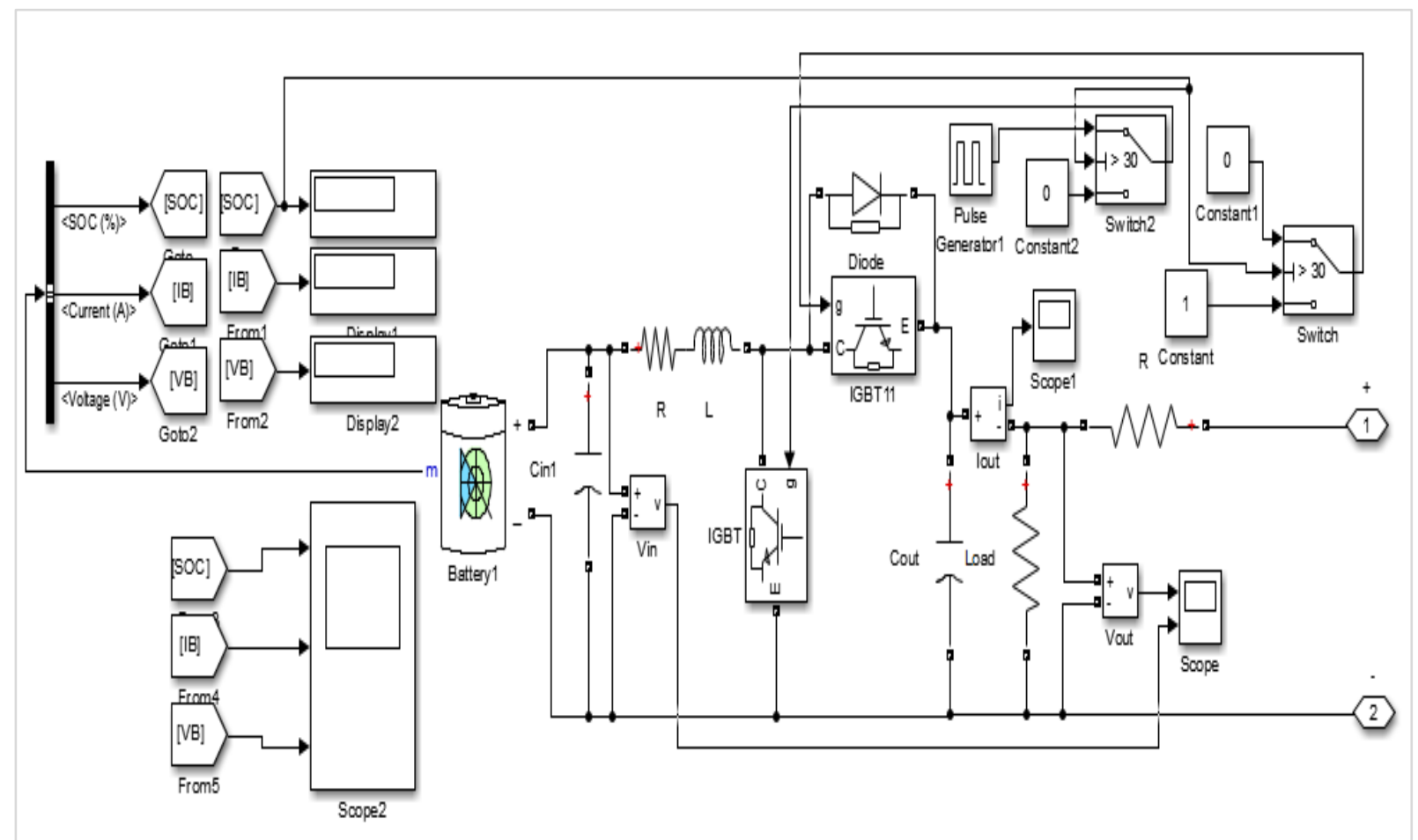

Figure 6 Simulation model for source 2- battery associated with DC microgrid across DC-DC bidirectional converter with SOC controller 
Dipti Patil et al.

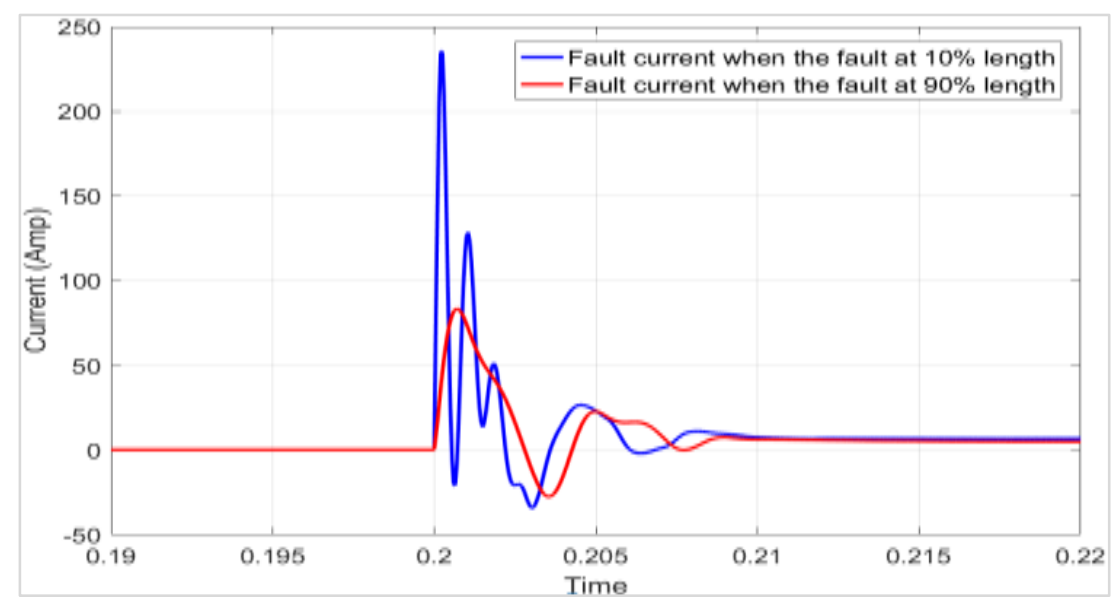

Figure 7 Oscillation in fault current in case of fault at $10 \%$ and $90 \%$ distance of the $1 \mathrm{~km}$ bus section

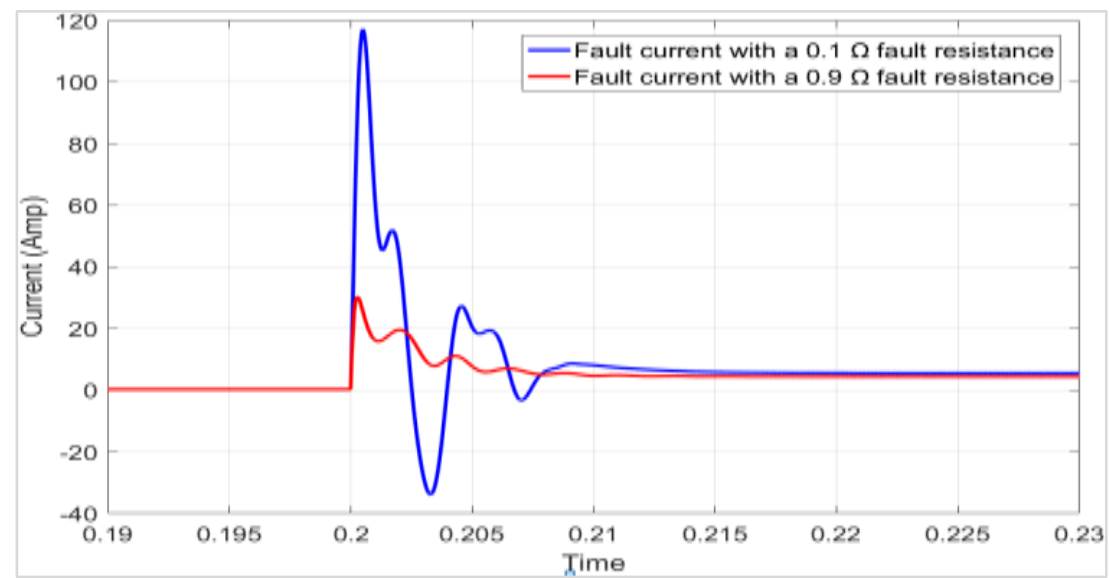

Figure 8 Oscillation in fault current in case of fault resistance of $0.1 \Omega$ and $0.9 \Omega$ at $50 \%$ distant of the $1-\mathrm{km}$ bus section

\section{Results}

4.1Simulation results and discussion

\subsubsection{Collection of data set}

The proposed method is first tested with the simulation model shown in Figure 4. Initially, the system is simulated under normal conditions. The data for the normal condition is collected and labeled as normal. The simulation time is set as $1 \mathrm{~s}$ and faults are initiated in between 0 to $1 \mathrm{~s}$. Bus to bus fault is initiated at each segment at $20 \%, 40 \%, 60 \%$, and $80 \%$ location with $0 \Omega, 0.1 \Omega, 0.4 \Omega, 0.6 \Omega$, and $0.8 \Omega$ fault resistances respectively, and fault types are labeled. Similarly, other abnormal conditions like overload, over-voltage, and light load are created by varying the load connected to the system and labeled accordingly. The system consists of 4 segments. So, bus to bus fault at 4 locations with five different resistances on each segment gives a total of 80 $(4 \times 4 \times 5)$ fault types. Also, considering normal, overload, over-voltage, light load conditions, a total of 84 parameters constitute the column of stored data. The rows are 1000 samples depending on simulation time, which is kept at $1 \mathrm{~s}$ and the sampling frequency is $1 \mathrm{kHz}$. The bus to bus fault is set at $0.2 \mathrm{~s}$ on the DC bus segment 2 (Figure 3) between a PV source and a battery source. The surge in segment fault current and the difference in positive/negative value are shown in Figure 9. Figures 10, 11, and Figure 12 shows overload, under load, and overvoltage conditions. Overload and light load conditions are created by applying twice and half the rated value of the load. Overvoltage is considered with a switching condition.

These abnormal conditions create similar oscillation as that of fault situations, hence it is important to learn the features of each condition. The proposed protection scheme learns the characteristics of normal, abnormal, and fault condition and is able to differentiate the system conditions. 


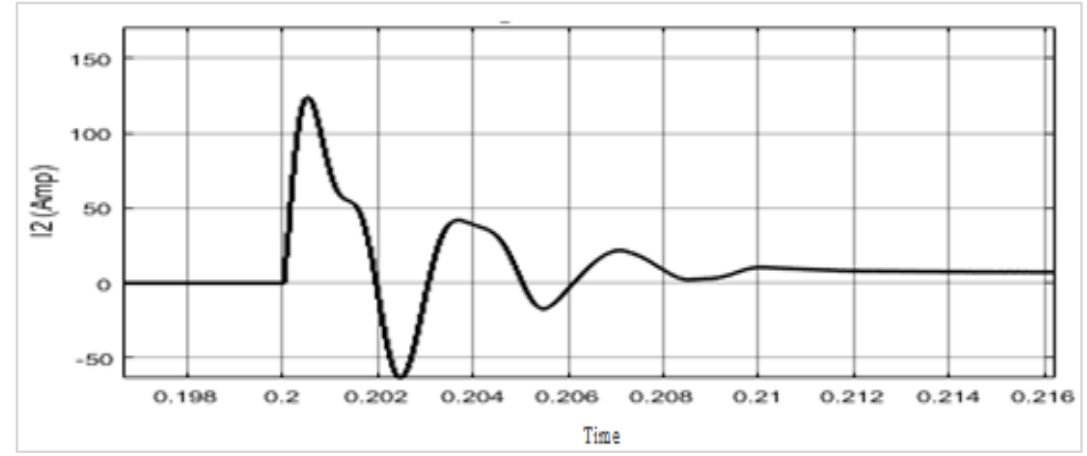

Figure 9 Segment 2 fault current due to bus to bus fault

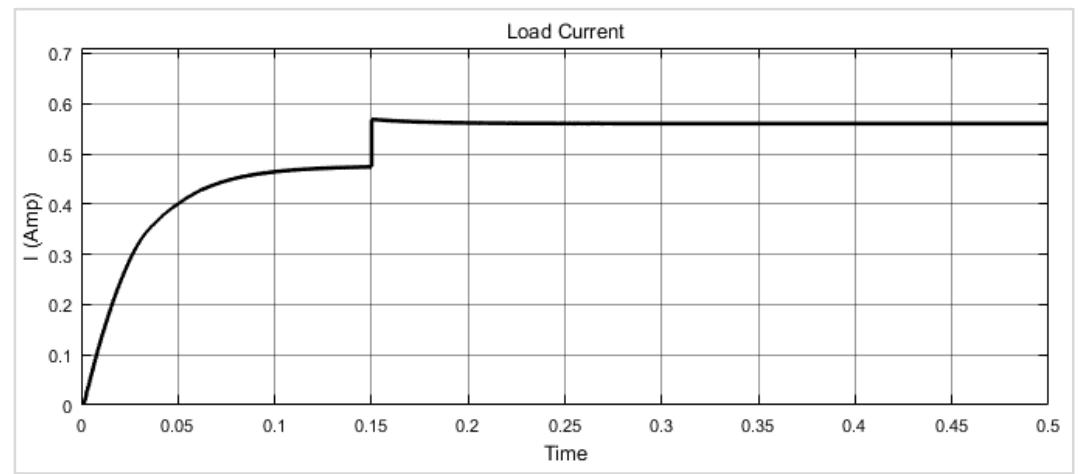

Figure 10 System overload condition

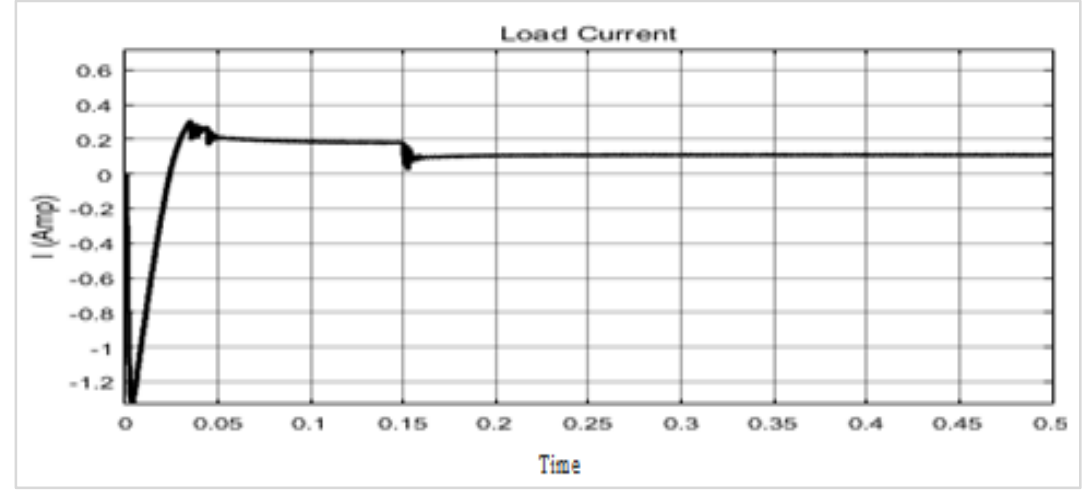

Figure 11 System light load condition

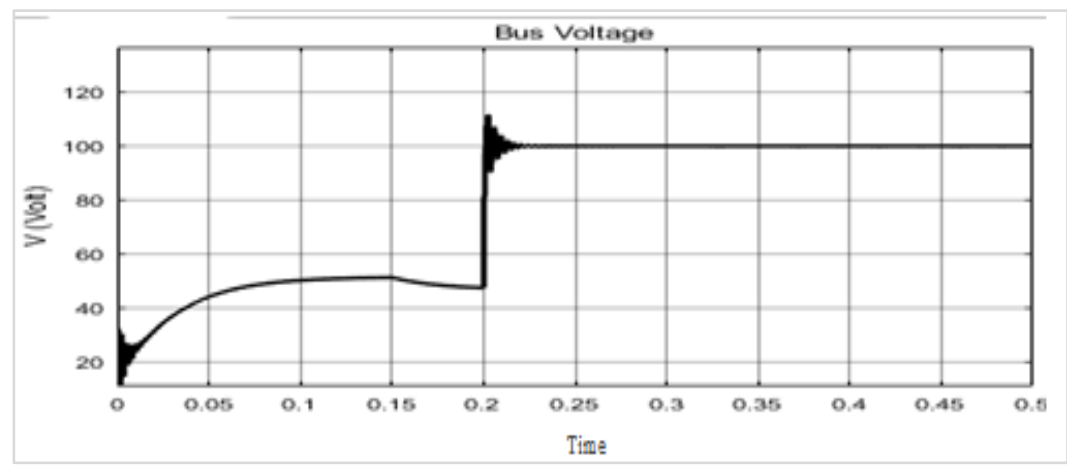

Figure 12 System overvoltage condition 


\subsection{Data augmentation and pre-processing}

The system is simulated for all the stated conditions 100 times. Ten different signals are generated from each signal by adding random noise of zero-mean Gaussian distribution. 2nd and 3rd order smoothing and sharpening are used to increase the generated signals further by 4 times. After data augmentation the total data set is collected is $84 \mathrm{X} 100 \mathrm{X} 10 \mathrm{x} 4=3$, 36,000 . Since the size of the data is large, it needs to carry out pre-processing i.e. PCA. The dimensions of data measurements at a time from the system are (5x1000) which can be split as 4 segment currents and one load current. (1x1000) dimensions for bus voltage measurement. Such huge dimension data require preprocessing. The measured segment current and bus voltage data are processed separately. The covariance matrix calculated from the collected data set then by using singular value decomposition is the principal components generated. After pre-processing dimensions are reduced to $(1 \times 1000)$ for measured current and $(1 \times 1000)$ for bus voltage. Since there are 84 measuring elements; the size of the dataset is reduced to $2 \times 84$. The pre-processing output is further used in the EMD for fault features extraction.

\subsection{Empirical mode decomposition of collected data}

EMD is applied to segment current and bus voltage separately. Fault first signal required normalizing the amplitude values to the range between zero and one (Equation 1).

$x_{\text {scaled }}=\frac{x-\min (x)}{\max (x)-\min (x)}$

Here, $\mathrm{x}$ represents a single feature/variable vector. The normalized signal is decomposed into $\mathrm{N}$ number of user-defined IMF components using EMD, here in this study 4 components and one residue component are considered as shown in Figure 13. The figure shows the IMFs decomposition of the fault signal in the time domain and the same length as the original signal and allows the preservation of varying frequency in time. Obtaining IMF of the real-time signal is important because occurrence faults or abnormalities often have multiple causes and each of these causes may happen at a specific time interval.

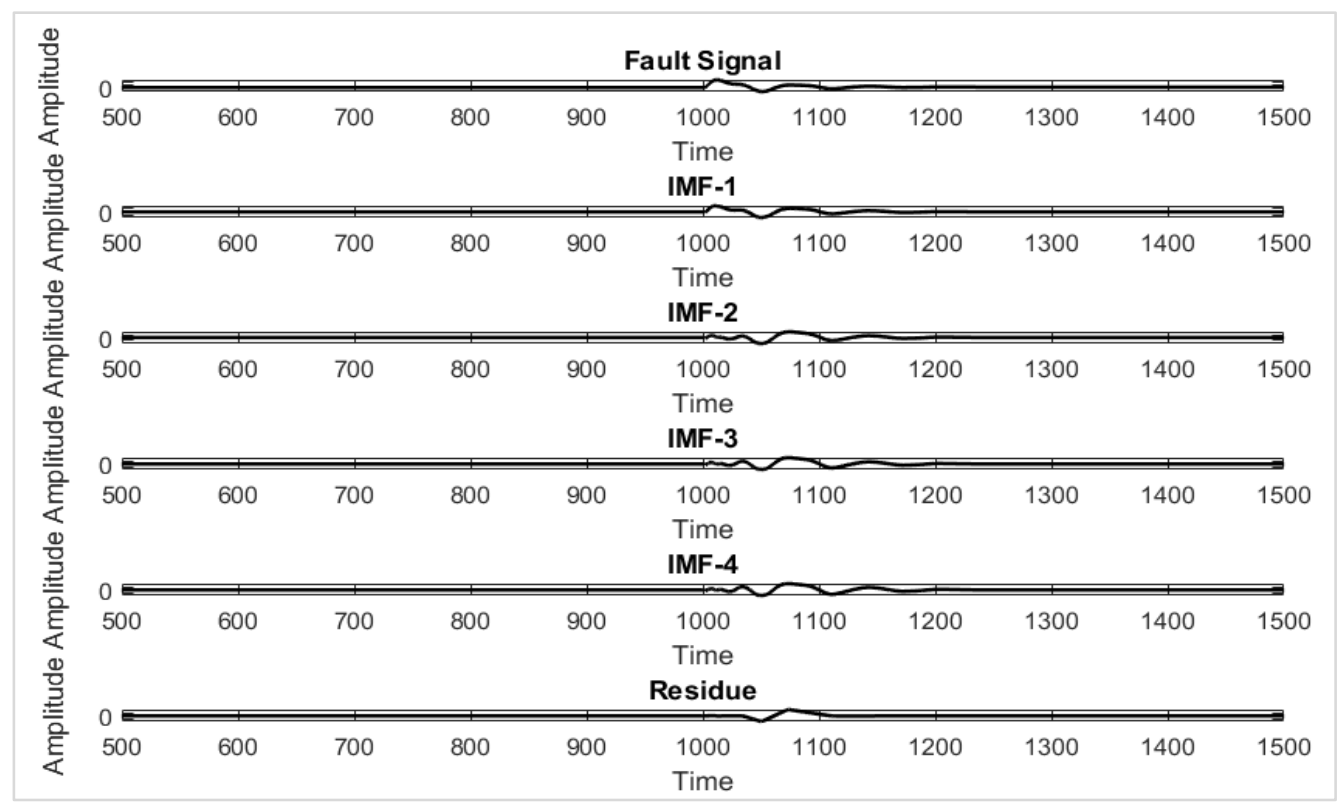

Figure 13 EMD of fault signal at segment 2(Figure 9) (first 4 IMFs and residue)

4.4Discrimination of fault type and abnormal conditions with the proposed CNN-based classifier

In the case of CNN, each IMF is fed as a separate channel, hence designed $\mathrm{CNN}$ is a multi-channel CNN with $\mathrm{N}$ channel input [27] as shown in Figure 14. In this case, 4 channels are used to feed the current signal and 4 channels are used to feed the 208 voltage signal. Three convolution layers were included and the quantities of filters in each layer were set as of 4-8-16 respectively. The convolution layers are trailed by a pooling layer. The activation technique rectified linear unit (ReLU) and soft-max function are used for the input, hidden layers, and output layer. The training is done with a stochastic gradient descent (SGD) optimizer and the loss 
function utilized was binary cross-entropy. The learning rate refreshing is finished after each 25 epochs and a half for transformation to the global minimum. The last layer is the dense layer in which the output is broken down into types of class. The quantity of output of CNN is identical with the quantity of classes in which the info signals have been grouped. The network is trained with the collected data set and corresponding fault label.
Figure 15 shows the training workflow, wherein the input signals are decomposed using EMD and fed to the network along with the output labels (annotated by the user). During the training process, the loss function is minimized by initializing and modifying the weights of the neurons. The validation process is used to evaluate the trained model with a testing data set Figure 16.

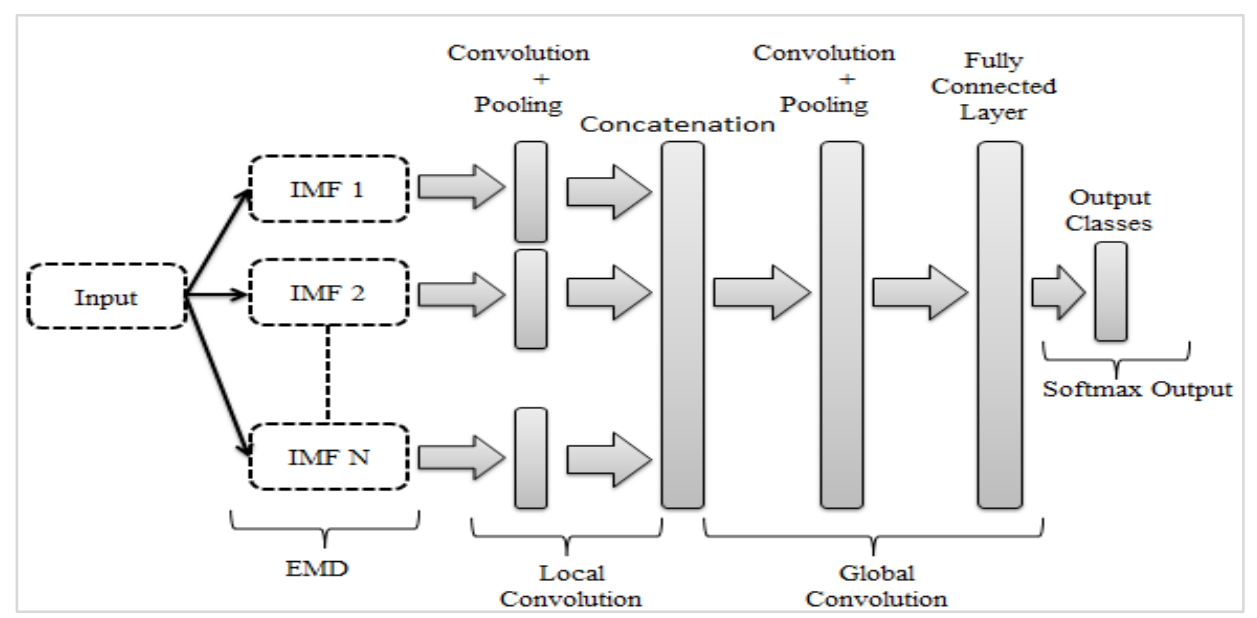

Figure 14 Multi-channel convolutional neural network

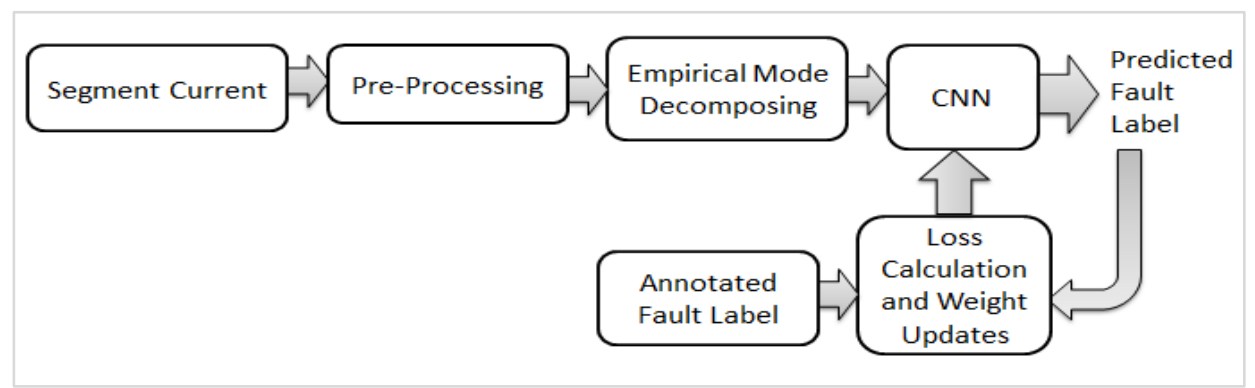

Figure 15 Training workflow

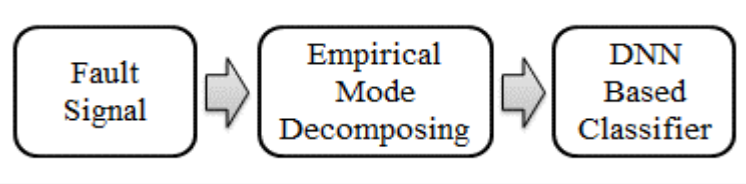

Figure 16 Inference workflow

The testing is done to assess the fault detection accuracy of the trained model so that the problem of overfitting can be avoided. The yield of the testing network is a vector wherein each value represents the probability of an information signal belonging to a certain class. The prepared model computes the weight probability of fault signal during validation. Now, depending on with which trained data the calculated weight probability of input fault signal 209 gets matched, fault type and location are recognized. The CNN model is trained with 100 iterations/epochs. Data is randomly divided into the normal, abnormal, and fault types cases into $75 \%$ data set are used for training and remaining $25 \%$ used for testing, which corresponds with the usual exercise [28]. The training /validation test break is similar for each run with an alternate irregular weight initialization. The computation is carried out on an exclusive CPU and the total time required for training was 10 hours. The trained record is ready as a hierarchical data format (HDF) document is stacked at the testing time in the network. Table 1 and Table 2 shows the outcomes for various types of fault recognition exactness for CNN. CNN model summary of the simulation model is shown in Figure 
Dipti Patil et al.

17. Overall statistics for $\mathrm{CNN}$ for simulation is shown in Table 3. The performance of the proposed classifier is analysed and evaluated based on various measures such as accuracy, specificity, and execution time. The accuracy is decided by taking the ratio of correct recognition to the total number of the input signal. Specificity is the ability of the classifier to identify negative results and execution time is the final time required to classify.
- The classification accuracy of the proposed scheme is $94.97 \%, 95 \%$ and $94 \%$ respectively for the fault types such as overload, overvoltage and light load.

- Specificity is 0.9994 .

- The execution time is $10 \mathrm{~ms}$.

Table 1 Fault detection accuracy in percentage for $20 \%$ and $40 \%$ location

\begin{tabular}{|c|c|c|c|c|c|c|c|c|c|c|}
\hline \multirow[t]{2}{*}{ Fault at } & \multicolumn{5}{|c|}{$\begin{array}{l}\text { Accuracy in } \% \text { in case of bus to bus fault at } \\
20 \% \text { location with fault resistance }(\Omega)\end{array}$} & \multicolumn{5}{|c|}{$\begin{array}{l}\text { Accuracy in } \% \text { in case of bus to bus fault at } \\
40 \% \text { location with fault resistance }(\Omega)\end{array}$} \\
\hline & 0 & 0.2 & 0.4 & 0.6 & 0.8 & 0 & 0.2 & 0.4 & 0.6 & 0.8 \\
\hline Segment 1 & 96 & 96 & 95 & 96 & 95 & 95 & 96 & 95 & 94 & 93 \\
\hline Segment 2 & 95 & 96 & 95 & 96 & 96 & 95 & 96 & 94 & 95 & 96 \\
\hline Segment 3 & 95 & 94 & 95 & 94 & 95 & 95 & 93 & 94 & 96 & 95 \\
\hline Segment 4 & 94 & 95 & 96 & 95 & 95 & 94 & 94 & 93 & 94 & 95 \\
\hline
\end{tabular}

Table 2 Fault detection accuracy in percentage for $60 \%$ and $80 \%$ location

\begin{tabular}{|c|c|c|c|c|c|c|c|c|c|c|}
\hline \multirow[t]{2}{*}{ Fault at } & \multicolumn{5}{|c|}{$\begin{array}{l}\text { Accuracy in } \% \text { in case of bus to bus fault at } \\
60 \% \text { location with fault resistance }(\Omega)\end{array}$} & \multicolumn{5}{|c|}{$\begin{array}{l}\text { Accuracy in \% in case of bus to bus fault at } \\
80 \% \text { location with fault resistance }(\Omega)\end{array}$} \\
\hline & 0 & 0.2 & 0.4 & 0.6 & 0.8 & 0 & 0.2 & 0.4 & 0.6 & 0.8 \\
\hline Segment 1 & 96 & 96 & 95 & 96 & 95 & 95 & 96 & 95 & 94 & 93 \\
\hline Segment 2 & 95 & 96 & 95 & 96 & 96 & 95 & 96 & 94 & 95 & 96 \\
\hline Segment 3 & 95 & 94 & 95 & 94 & 95 & 95 & 93 & 94 & 96 & 95 \\
\hline Segment 4 & 94 & 95 & 96 & 95 & 95 & 94 & 94 & 93 & 94 & 95 \\
\hline
\end{tabular}

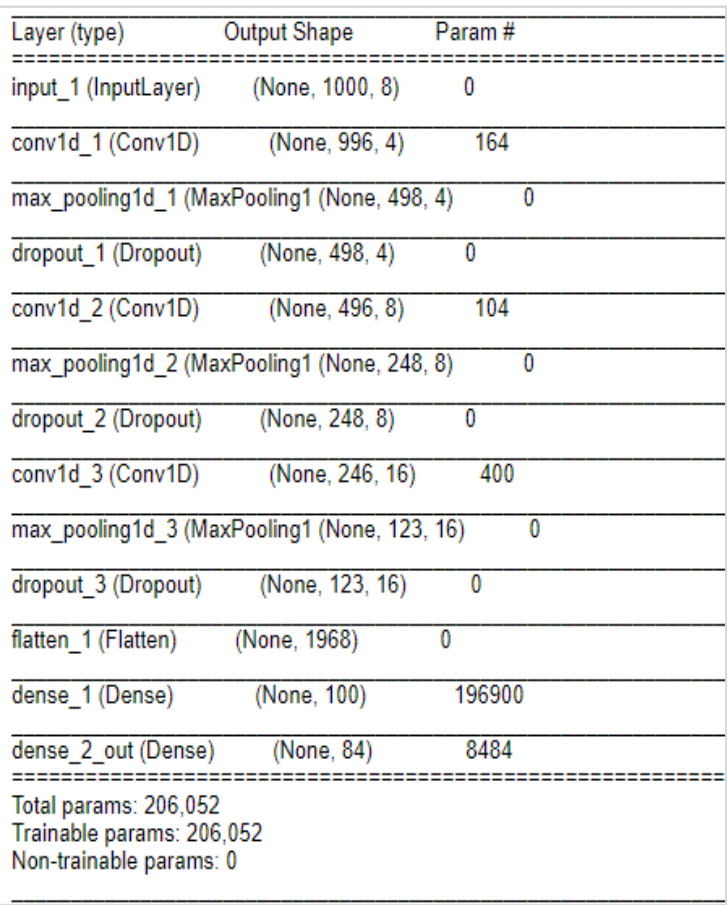

Figure 17 TensorFlow CNN model summary for simulation model
Table 3 Overall statistics for TensorFlow CNN for simulation model

\begin{tabular}{ll}
\hline Accuracy & 0.9497 \\
\hline Error & 0.0503 \\
\hline Sensitivity & 0.9497 \\
\hline Specificity & 0.9994 \\
\hline Precision & 0.9499 \\
\hline False Positive Rate & $6.0557 \mathrm{e}-04$ \\
\hline F1_score Correlation & 0.9498 \\
\hline $\begin{array}{l}\text { Matthews } \\
\text { Coefficient }\end{array}$ & 0.9492 \\
\hline Kappa & 0.5319 \\
\hline
\end{tabular}

The preprocessed dataset was also given as input to the Gaussian mixture model (GMM) and support vector machine (SVM) classifiers to compare the performance of the proposed CNN classifier in terms of accuracy, specificity, and execution time with their performance. GMM utilizes the weighted amount of a few Gaussian distribution functions to assess the probability density distribution of samples and the grouping result is to amplify the probability density of samples [29]. SVM is the learning method that utilizations input vectors to plan nonlinearly into an element space whose aspect is high [30]. The comparative results of these classifiers for the same dataset are shown in Figures 18 and 19. 
From the results, it is shown that the proposed protection scheme has higher accuracy, higher specificity, and low execution time with comparative existing classification methods in the discrimination of fault type and other abnormal situations.

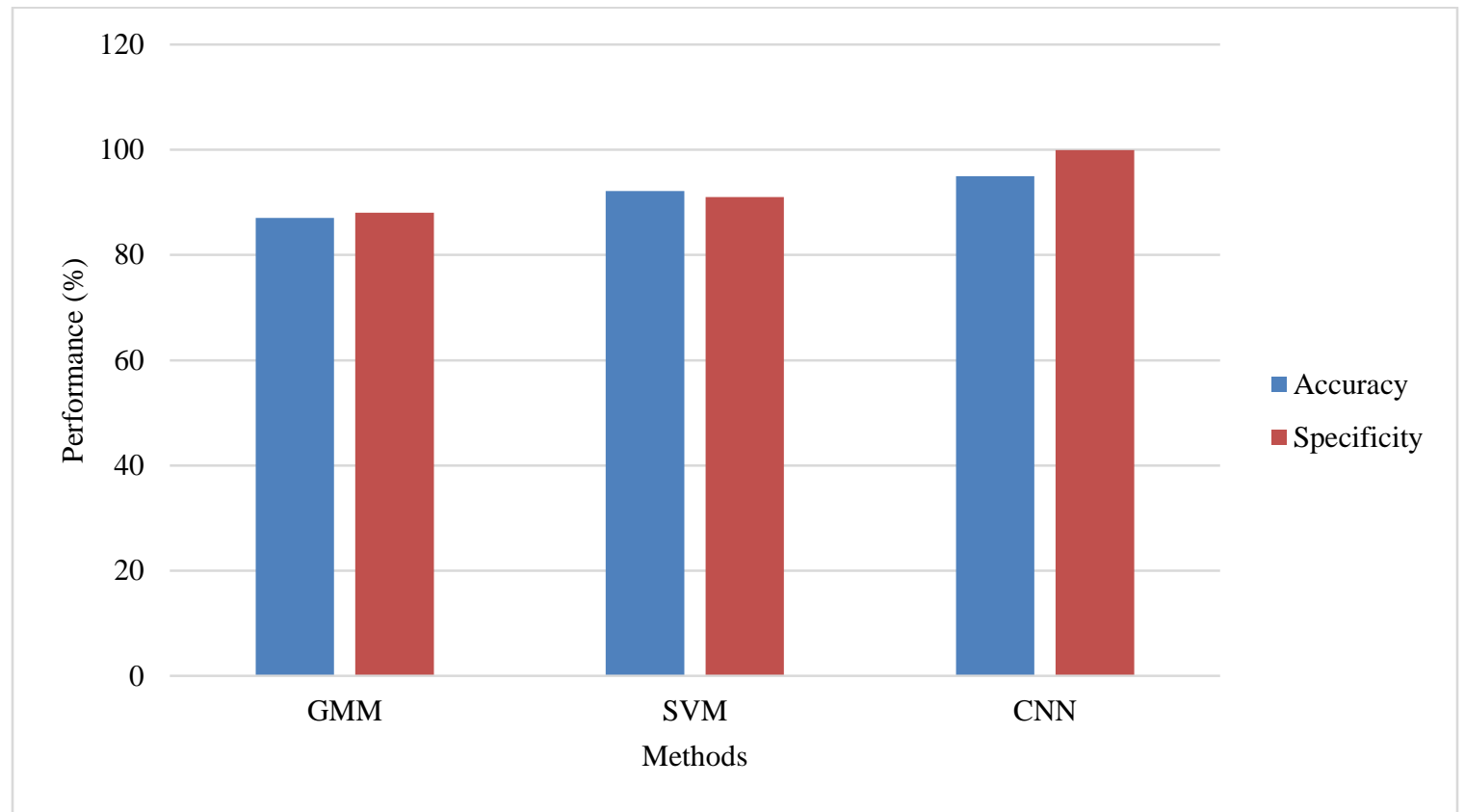

Figure 18 Performance comparison based on accuracy and specificity

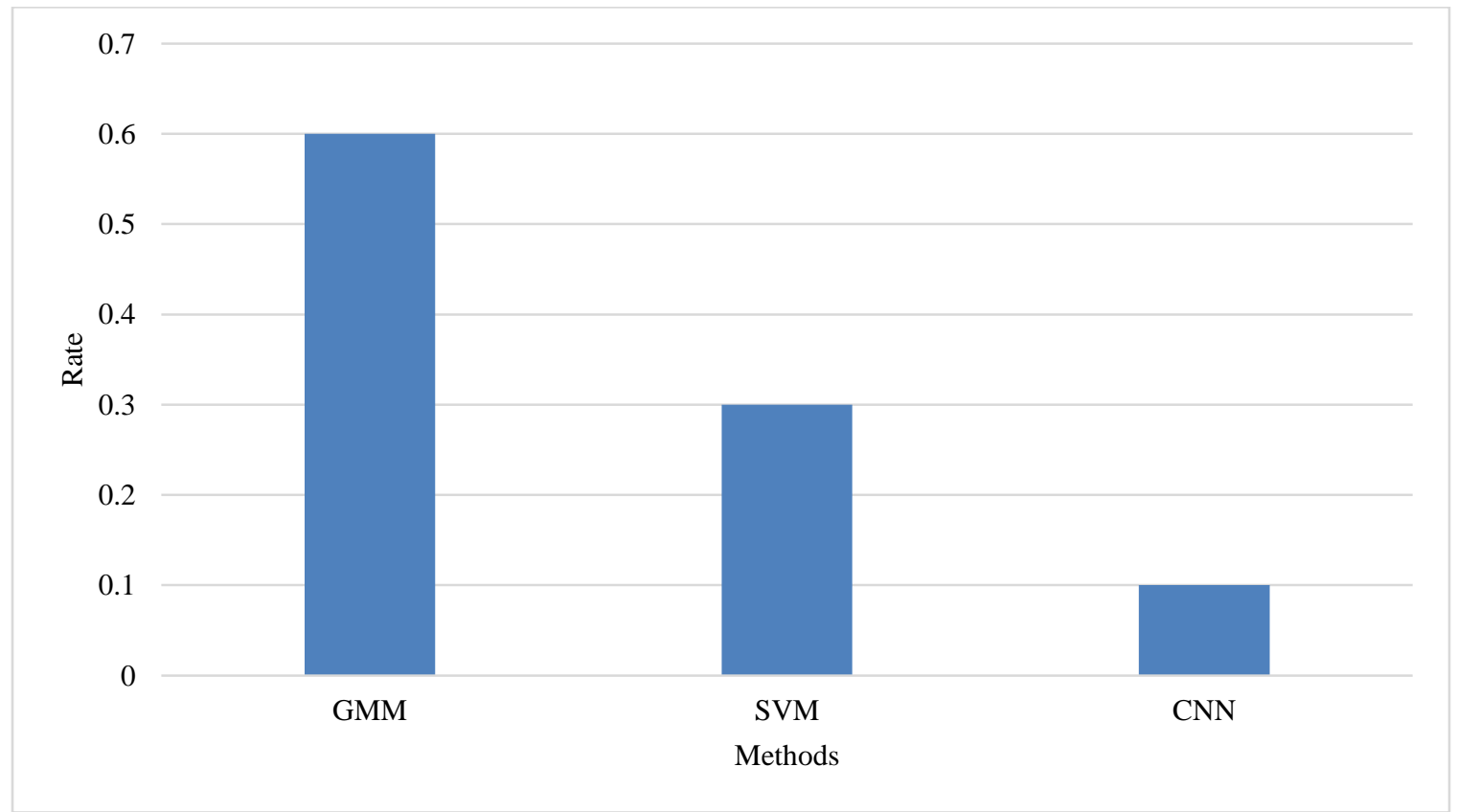

Figure 19 Execution time comparisons

\subsection{Experimental results of LVDC microgrid}

A 48-volt LVDC microgrid hardware prototype of the simulation model is developed. MPPT control has not been implemented for the hardware. So, the sole 211 purpose of providing a power electronics interface is to control voltage and current. The power flow is bidirectional as battery charges and discharges. Hence a bidirectional converter is the best fit for the 
application. Both converters are $1000 \mathrm{~W}$ and designed using $600 \mathrm{~V}, 100 \mathrm{~A}$ insulated-gate bipolar transistor (IGBT) modules with an operating frequency of $20 \mathrm{kHz}$. A PI controller has been implemented in the converter to control either voltage or current. The power switches are provided at each end of the bus segment to segregate the faulty segment. The microgrid load is also integrated into the microgrid bus utilizing the power switch. All these converters and power switches are operated by giving pulse width modulation (PWM) signals from the TMS320F28069 digital signal processor (DSP) [31].

The proposed method is tested on a hardware model to validate the simulation results. The circuit is tested for the bus to bus fault without any fault resistance and bus to bus fault with $0.8 \Omega$ resistances is considered. Both faults are initiated on $20 \%$ and $60 \%$ locations. Other abnormal conditions are created like overload, over-voltage, and light load by varying the connected load. Each case is initiated at least 10 times. Data augmentation preprocessing training and testing are similar to the simulation model.

The bus-to-bus fault at segment 1 at a distance of $60 \%$ from the source is shown in Figure 20. All the sources operated with limited capacity to ensure the safety of switches. The fault current shows noise due to the effect of measuring devices and the communication channels used in the system. So, by decomposing fault signals using EMD the correct fault information in the frequency domain can get extracted. The decomposition of the fault signal is shown in Figure 21. The fault detection accuracy of the design CNN for hardware is shown in Table 4. The network also shows the detection accuracy for overload 92\%, over-voltage 93\%, and light load 93\% in the case of hardware.

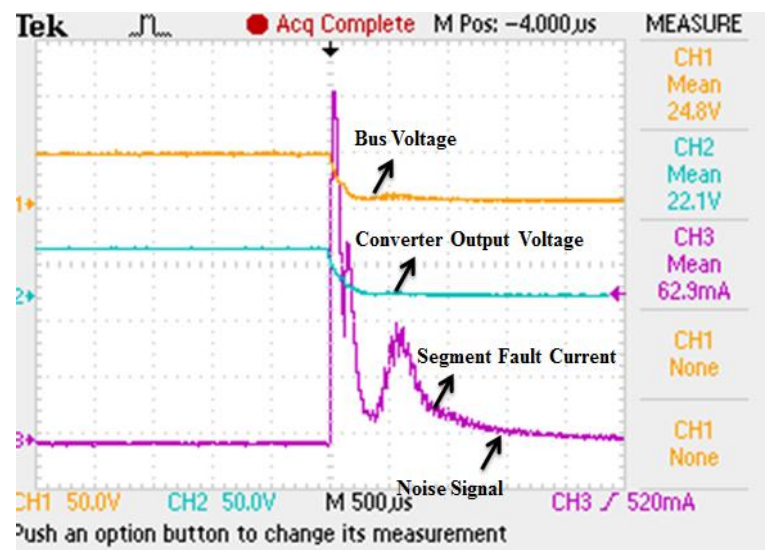

Figure 20 Bus to bus fault current at segment 1

212

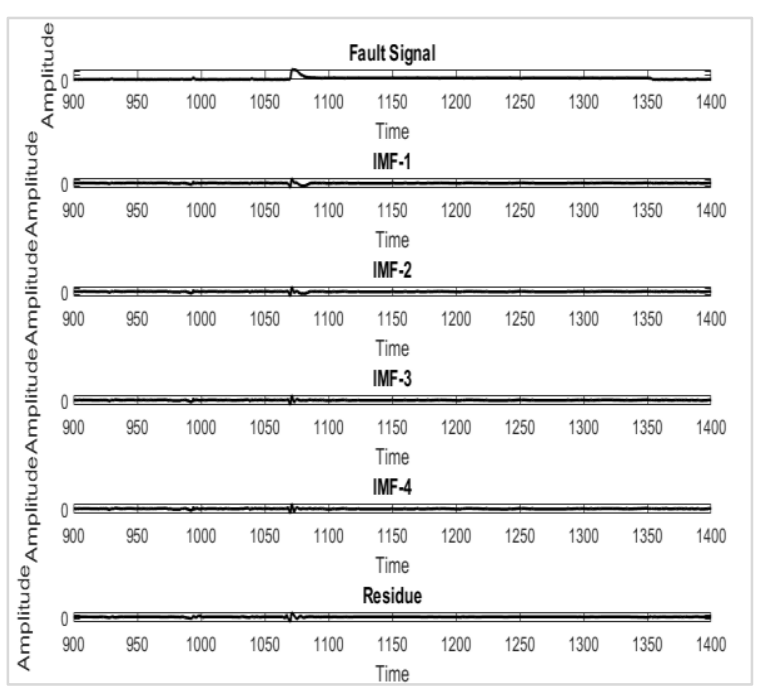

Figure 21 EMD of fault signal segment 1(Figure 22) (first 4 IMFs and residue)

Table 4 Accuracy in percentage for fault detection of hardware model

\begin{tabular}{lllll}
\hline Fault at & $\begin{array}{l}\text { \% Accuracy for } \\
\text { Bus to bus fault at } \\
\mathbf{2 0 \%} \text { location with } \\
\text { fault } \\
(\boldsymbol{\Omega})\end{array}$ & $\begin{array}{l}\text { resistance } \\
\text { Bus to bus fault at } \\
\end{array}$ & $\begin{array}{l}\mathbf{6 0 \%} \text { location with } \\
\text { fault resistance } \\
(\boldsymbol{\Omega})\end{array}$ \\
\cline { 2 - 5 } & 0 & 0.8 & 0 & 0.8 \\
\hline Segment 1 & 88 & 91 & 89 & 91 \\
\hline Segment 2 & 92 & 90 & 91 & 91 \\
\hline Segment 3 & 90 & 91 & 87 & 92 \\
\hline Segment 4 & 88 & 86 & 93 & 90 \\
\hline
\end{tabular}

Overall statistics for TensorFlow $\mathrm{CNN}$ for hardware is shown below in Table 5 and CNN model summary shown in Figure 22.

Table 5 Overall statistics for TensorFlow CNN for hardware

\begin{tabular}{ll}
\hline Accuracy & 0.9060 \\
\hline Error & 0.0940 \\
\hline Sensitivity & 0.9061 \\
\hline Specificity & 0.9951 \\
\hline Precision & 0.9065 \\
\hline False Positive Rate & 0.0049 \\
\hline F1_score & 0.9061 \\
\hline $\begin{array}{l}\text { Matthews Correlation } \\
\text { Coefficient }\end{array}$ & 0.9013 \\
\hline Kappa & 0.0110 \\
\hline
\end{tabular}




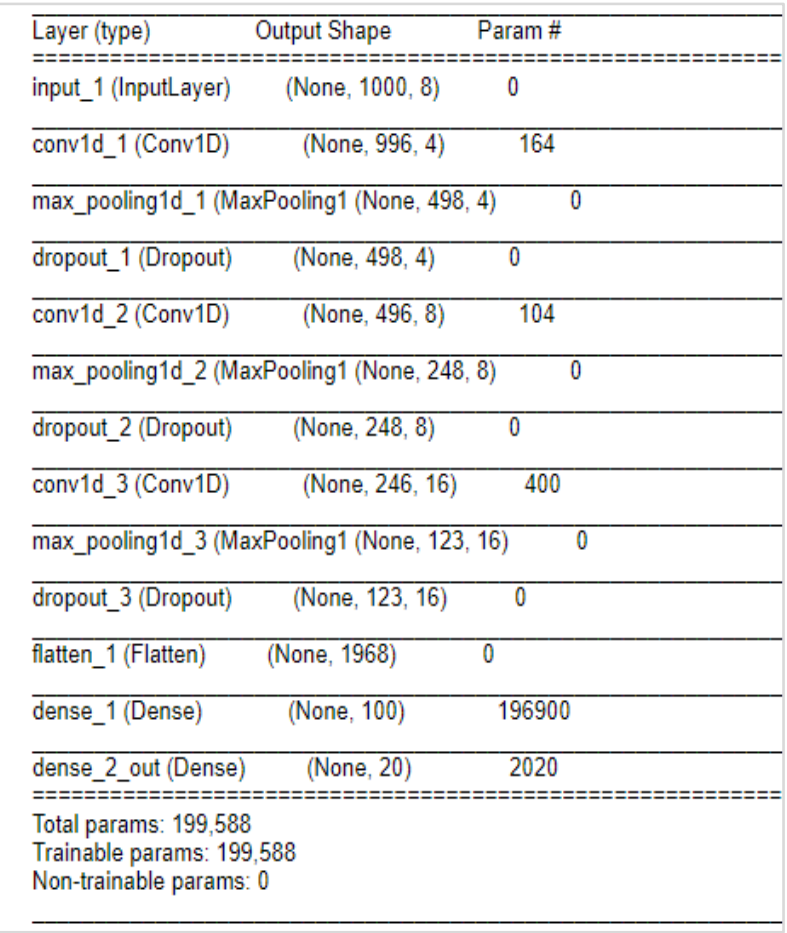

Figure 22 TensorFlow CNN model for hardware summary

\section{Discussion and limitations}

From the simulation and hardware results, it can be seen that the empirical mode decomposition method extracts low-frequency structures and represents a signal like the multi-resolution analysis. EMD allows the data to be examined in an adaptive timefrequency-amplitude space for non-stationary signals. It decomposes the input signal into a userdefined number of IMF. The DNN method can be performed with all types of features and can extract characteristics independently. However, feeding the pre-extracted features to CNN's further improves the detection accuracy and also simplifies the network complexity.

The performance of the proposed classifier is analyzed and evaluated by measures such as accuracy, specificity, and execution rate. Figure 18 shows the comparison of classification accuracy of the proposed with the SVM and GMM classification methods. The proposed classifier achieved an accuracy of $94.97 \%$, whereas SVM achieved $92.15 \%$ and GMM achieved $87.02 \%$ accuracy. Figures 18 and 19 show the comparison of the proposed method with other classification methods in terms of specificity and execution rate respectively. The proposed classifier has a specificity of 0.95 whereas SVM has 0.91 and GMM has 0.88. The execution 213 rate required for the proposed method is less than SVM and GMM classifiers. The model summary of TensorFlow design for simulation and hardware is shown in Figure 17 and Figure 22. The model summary depicts the CNN layer design with various layer types and sizes. The exceptional performance of the proposed method through simulation and hardware model is presented in Table III and Table V in terms of overall statistics. Thus, we can conclude that the proposed method is proven to be better compared to other existing methods for the addressed problem. Still, detailed mathematical analysis needs to be carried out to reach the desired decomposition level for EMD which will require distinguishing the fault features. The network complexity can be evaluated by varying the number of layers in $\mathrm{CNN}$ for optimal results. A complete list of abbreviations is shown in Appendix I.

\section{Conclusion and future scope}

This paper proposed an advanced method for fault detection and localization of LVDC microgrid using EMD as feature extractor and DNN as the classifier. The simulation model is developed to extract measurements for computation to the classifier. DC segment currents signal by initiating fault at a different location with different fault resistances and DC bus voltage are used to train and test the $\mathrm{CNN}$ network. Classifier identifies the fault type and location accurately. The proposed classifier accuracy, specificity and execution time is compared with GMM and SVM classifier to show the effectiveness of the proposed scheme. Simulation results are also validated by hardware results of the $48 \mathrm{~V}$ DC microgrid. The results indicate that the suggested technique can produce more precise fault detection and fault type classification. The proposed method also detects abnormalities like overload, overvoltage, and light load conditions even under noisy environments. Consequently, the DNN based strategy can be viewed as an exceptionally proficient method for LVDC microgrids in fault detection and localization.

Future exploration endeavors will be given to:

1. The recognition of the most competent empirical mode decomposition level still fit for identification of a wide range of fault classes.

2. Identification of the numbers of layers in $\mathrm{CNN}$, which will give the classification accuracy maximum.

Acknowledgment

None. 
Conflicts of interest

The authors have no conflicts of interest to declare.

\section{Authors contribution statement}

Dipti Patil: Methodology, simulation model development, hardware design development, analysis, interpretation of results, paper writing. Bindu S.: Interpretation of results, review and editing. Sushil Thale: Hardware system design, interpretation of results, review and editing.

\section{References}

[1] Shuai Z, Fang J, Ning F, Shen ZJ. Hierarchical structure and bus voltage control of DC microgrid. Renewable and Sustainable Energy Reviews. 2018; 82:3670-82.

[2] Zia MF, Elbouchikhi E, Benbouzid M. Microgrids energy management systems: a critical review on methods, solutions, and prospects. Applied Energy. 2018; 222:1033-55.

[3] Javed W, Chen D. Low voltage DC microgrid protection system-a review. In international universities power engineering conference (UPEC) 2018 (pp. 1-6). IEEE.

[4] Beheshtaein S, Cuzner R, Savaghebi M, Guerrero JM. Review on microgrids protection. IET Generation, Transmission \& Distribution. 2019; 13(6):743-59.

[5] Gottwalt S, Gärttner J, Schmeck H, Weinhardt C. Modeling and valuation of residential demand flexibility for renewable energy integration. IEEE Transactions on Smart Grid. 2016; 8(6):2565-74.

[6] Meng L, Shafiee Q, Trecate GF, Karimi H, Fulwani D, $\mathrm{Lu} \mathrm{X}$, et al. Review on control of DC microgrids and multiple microgrid clusters. IEEE Journal of Emerging and Selected Topics in Power Electronics. 2017; 5(3):928-48.

[7] Javed W, Chen D, Farrag ME, Xu Y. System configuration, fault detection, location, isolation and restoration: a review on LVDC microgrid protections. Energies. 2019; 12(6):1-30.

[8] Montoya R, Poudel BP, Bidram A, Reno MJ. DC microgrid fault detection using multiresolution analysis of traveling waves. International Journal of Electrical Power \& Energy Systems. 2022.

[9] Gaurav S, Nougain V, Panigrahi BK. Protection of low-voltage DC microgrid based on series $\mathrm{R}-\mathrm{L}-\mathrm{C}$ equivalent circuit utilising local measurements. IET Generation, Transmission \& Distribution. 2020; 14(18):3877-85.

[10] Li M, Zhang D, Lu S, Tang X, Phung T. Differential evolution-based overcurrent protection for DC microgrids. Energies. 2021; 14(16):1-19.

[11] Eslami R, Hosseini SA. Presenting new triple methods for fault detection, location, and its identification in DC microgrid. Iranian Journal of Science and Technology, Transactions of Electrical Engineering. 2020; 44(2):849-60.

[12] Jayamaha DK, Lidula NW, Rajapakse AD. Waveletmulti resolution analysis based ANN architecture for fault detection and localization in DC microgrids. IEEE Access. 2019; 7:145371-84.
[13] Leh NA, Zain FM, Muhammad Z, Abd HS, Rosli AD. Fault detection method using ANN for power transmission line. In 10th IEEE international conference on control system, computing and engineering (ICCSCE) 2020 (pp. 79-84). IEEE.

[14] Wang Z, Xu L. Fault detection of the power system based on the chaotic neural network and wavelet transform. Complexity. 2020.

[15] Ashok V, Yadav A. A real-time fault detection and classification algorithm for transmission line faults based on MODWT during power swing. International Transactions on Electrical Energy Systems. 2020; 30(1).

[16] Paul M, Debnath S. Fault detection and classification scheme for transmission lines connecting windfarm using single end impedance. IETE Journal of Research. 2021:1-13.

[17] Mikołajczyk A, Grochowski M. Data augmentation for improving deep learning in image classification problem. In international interdisciplinary $\mathrm{PhD}$ workshop (IIPhDW) 2018 (pp. 117-22). IEEE.

[18] Wang Y, Ma X, Qian P. Wind turbine fault detection and identification through PCA-based optimal variable selection. IEEE Transactions on Sustainable Energy. 2018; 9(4):1627-35.

[19] Jarrahi MA, Samet H, Sahebi A. An EMD based fault type identification scheme in transmission line. In 24th Iranian conference on electrical engineering (ICEE) 2016 (pp. 422-7). IEEE.

[20] Wang L, Liu H, Dai LV, Liu Y. Novel method for identifying fault location of mixed lines. Energies. 2018; 11(6):1-19.

[21] Eren L, Ince T, Kiranyaz S. A generic intelligent bearing fault diagnosis system using compact adaptive 1D CNN classifier. Journal of Signal Processing Systems. 2019; 91(2):179-89.

[22] Ozcan IH, Devecioglu OC, Ince T, Eren L, Askar M. Enhanced bearing fault detection using multichannel, multilevel 1D CNN classifier. Electrical Engineering. 2021:1-13.

[23] Patil DD, Bindu S. Real time protection technique for DC microgrid using local measurements. In technologies for smart-city energy security and power (ICSESP) 2018 (pp. 1-6). IEEE.

[24] Aemro YB, Moura P, De AAT. Design and modeling of a standalone DC-microgrid for off-grid schools in rural areas of developing countries. Energies. 2020; 13(23):1-24.

[25] Patil C, Thale S, Muchande S, Kadam AH. A novel protection scheme for DC microgrid with hierarchical control. In international conference on smart energy grid engineering (SEGE) 2017 (pp. 117-22). IEEE.

[26] Shamsoddini M, Vahidi B, Razani R, Mohamed YA. A novel protection scheme for low voltage DC microgrid using inductance estimation. International Journal of Electrical Power \& Energy Systems. 2020.

[27] Srinivasamurthy RS. Understanding 1D convolutional neural networks using multiclass time-varying signals. Doctoral Dissertation, Clemson University. 
[28] Srivastava N, Hinton G, Krizhevsky A, Sutskever I, Salakhutdinov R. Dropout: a simple way to prevent neural networks from overfitting. The Journal of Machine Learning Research. 2014; 15(1):1929-58.

[29] Shi J, He Q, Wang Z. GMM clustering-based decision trees considering fault rate and cluster validity for analog circuit fault diagnosis. IEEE Access. 2019; 7:140637-50.

[30] Ray P, Mishra DP. Support vector machine based fault classification and location of a long transmission line. Engineering Science and Technology, an International Journal. 2016; 19(3):1368-80.

[31] Som S, Samantaray SR. Efficient protection scheme for low-voltage DC micro-grid. IET Generation, Transmission \& Distribution. 2018; 12(13):3322-9.

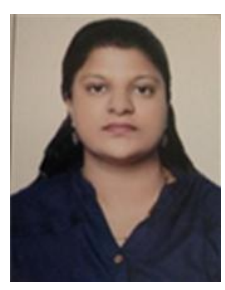

Dipti Patil received her B.E. degree in Electrical Engineering from Government College of Engineering, Amaravati, India, and M. E. degree in Electrical Power System from Veermata Jijabai Technological Institute, Mumbai, India. She is currently working towards Ph.D. degree in the field of electrical engineering at the Fr. C. Rodrigues Institute of Tech., Navi Mumbai, India. Her area of research includes Power system, Renewable Energy Systems, and Microgrids.

Email: diptipatil009@gmail.com

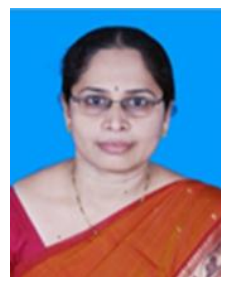

Dr. Bindu $\mathbf{S}$ was born in Kerala state, in India, in June 1970. After graduating in Electrical and Electronics Engineering in 1992 she did her Masters in Power System from Mumbai University in 2001. She did Ph.D in 2015 from Mumbai University. She has been with Fr C Rodrigues Institute of Technology for the last 27 years and is currently Professor and Head of the Department of the Electrical Engineering Department. Her research interest is in the areas of Power Systems and High Voltage Engineering.

Email: bindu.s@fcrit.ac.in

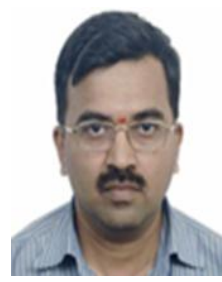

Dr. Sushil Thale obtained his Bachelor's degree in Electrical Engg. in 1992 and M.E. in Electrical Engg in 1996 from University of Mumbai, India. He completed his Ph.D. from Indian Institute of Technology-Bombay with specialization in Power Electronics and Power System in 2015. $\mathrm{He}$ is currently working as Professor (Electrical) and Dean (R\&D) at Fr. C. Rodrigues Institute of Tech., Navi Mumbai. His areas of research include Renewable Energy Systems, Electric Vehicles control, and Microgrids.

Email: sushil.thale@ fcrit.ac.in

\begin{tabular}{lll}
\multicolumn{2}{l}{ Appendix I } \\
\hline S. No. & Abbreviation & Description \\
\hline 1 & AC & Alternating Current \\
\hline 2 & AI & Artificial Intelligence \\
\hline 3 & CNN & Convolutional Neural Network \\
\hline 4 & DC & Direct Current \\
\hline 5 & DNN & Deep Neural Network \\
\hline 6 & DSP & Digital Signal Processors \\
\hline 7 & DWT & Discrete Wavelet Transform \\
\hline 8 & EMD & Empirical Mode Decomposition \\
\hline 9 & ESS & Energy Storage System \\
\hline 10 & GMM & Gaussian Mixture Model \\
\hline 11 & IGBT & Insulated-gate Bipolar Transistor \\
\hline 12 & IMF & Intrinsic Mode Functions \\
\hline 13 & LVDC & Low Voltage Direct Current \\
\hline 14 & MODWT & Maximal Overlap Discrete Wavelet \\
& & Transform \\
\hline 15 & MPPT & Maximum Power Point Tracking \\
\hline 16 & PCA & Principal Component Analysis \\
\hline 17 & PV & Photovoltaic \\
\hline 18 & PWM & Pulse Width Modulation \\
\hline 19 & ReLU & Rectified Linear Unit \\
\hline 20 & SGD & Stochastic Gradient Descent \\
\hline 21 & SOC & State of Charge \\
\hline 22 & SVM & Support Vector Machine \\
\hline 23 & VSC & Voltage Source Converter \\
\hline & &
\end{tabular}

